\title{
CRESCIMENTO E DIFERENCIAÇÃO "IN VITRO" DE CEPAS DE TRYPANOSOMA CRUZI, ISOLADA DE ANIMAIS SILVESTRES *
}

Dalva A. Mello **

Monamaris $M$. Borges ***

Lúcia Helena Chiarini ****

\begin{abstract}
RSPUB9/531
MEl.lo, D. A. et al. Crescimento e diferenciação "In vitro" de cepas de Trypanosoma cruzi, isoladas de animais silvestres. Rev. Saúde públ., S. Paulo, 14: $569-81,1980$.

Resumo: Foram estudadas três cepas de $\mathrm{T}$. cruzi isoladas de Didelphis albiventris (R52, R64 e R65) e uma isolada de Calomys callosus (M226), quanto ao comportamento "In vitro" no meio LIT. A evolução da população dos tripanossomas com relação ao crescimento e morfogênese foi acompanhada por um periodo de 13 dias ( 312 horas), em intervalos regulares. As contagens diferenciais, separando-se formas amastigotas, epimastigotas e tripomastigotas, foram realizadas na câmara de Neubauer. Os resultados obtidos levaram à conclusão de que as 4 cepas estudadas têm comportamento distintos. O melhor crescimento obtido ocorreu para a cepa M226, seguindo-se por ordem decrescente a R65, R52 e R64. Os picos das populaşóes ocorreram como segue: M226, entre 192-264 horas; R65 entre 168-240 horas; R52 às 240 horas $e$ R64 entre 264-312 horas.
\end{abstract}

UNITERmos Trypanosoma cruzi, cepas silvestres. Didelphis albiventris. Calomys callosus.

\section{INTRODUÇÃO}

Chagas 8 (1909), foi o primeiro autor a verificar morfogênese do Tripanosoma cruzi em meio de cultura acelular. Posteriormente, numerosos trabalhos foram realizados sobre cultivo de $T$. cruzi "In vitro" em diferentes meios acelulares, sendo analisados vários aspectos do comportamento deste protozoário. Entre outros trabalhos sobre estes assuntos, chama atenção os de: Muniz e
Freitas ${ }^{11}$ (1946), Noble 12 (1955), Pick ${ }^{15}$ (1959), Camargo 6 (1964), Brener e Chiari 4 (1965), Castellani e col..$^{i}$ (1967), Pan ${ }^{14}$ (1971), Chiari ${ }^{9}$ (1974).

No que se refere particularmente a estudos com cepas de $T$. cruzi isoladas de animais silvestres salientam-se os trabalhos de Norman e col.13 (1959) e de Ribeiro e Barretto ${ }^{16}$ (1975). O último autor tentou

* Trabalho realizado com auxílio do Conselho Nacional de Desenvolvimento Científico e Tecnológico (CNPq - PDE/02-1-06, FUB-CNPq, cadastro ne 81.961).

* Do Departamento de Medicina Complementar da Faculdade de Ciências da Saúde da Universidade de Brasilia - 70910 - Brasilia, DF - Brasil.

** Bolsista do CNPq

*** Do Departamento de Estatística do Instituto de Ciências Exatas da Universidade de Brasília - 70910 - Brasília, DF - Brasil. 
MELLO, D. A. et al. Crescimento e diferenciação "In vitro" de cepas de Trypanosoma cruzi, isoladas de animais silvestres. Rev. Saude públ., S. Paulo, 14:569-81, 1980.

relacionar o tipo de crescimento de cepas de $T$. cruzi, cultivadas no meio Warren, com o comportamento em animais e polimorfismo das formas sanguícolas baseandose nas observações realizadas por Brener ${ }^{2.3}$ (1965 e 1977), Brener e Chiari 5 (1963) e Andrade ${ }^{1}$ (1976).

Com o objetivo de contribuir acrescentando novos dados experimentais ao assunto, este trabalho apresenta resultados obtidos sobre crescimento e diferenciação "In vitro" de cepas de T. cruzi, isoladas de animais silvestres.

\section{MATERIAL E METODOS}

As cepas utilizadas neste trabalho tiveram as seguintes origens: R52 foi isolada de uma fêmea de Didelphis albiventris (Marsupialia) capturada no norte do município de Formosa, Goiás, em 17 de setembro de 1975; R64 foi isolada também de uma fêmea de $D$. albiventris capturada no município de Mambai, Goiás, em 20 de setembro de 1975; R65 teve a mesma origem, data de captura e procedência da R52; M226 foi isolada de uma fêmea de Calomys callosus (Rodentia) coletada também no norte do municipio de Formosa em 10 de dezembro de 1975. Estas cepas eram mantidas em camundongos albinos e em $C$. callosus nascidos em laboratório, através de inoculaçōes com fezes positivas de barbeiros alimentados nestes animais.

Para os isolamentos em meio de cultura acelular e para os estudos sobre o crescimento e diferenciação dos tripanossomas, foi utilizado o meio LIT. O volume do meio de cultura em cada tubo foi de $8 \mathrm{ml}$. A proporção de antibiótico por tubo com LIT foi de $0,8 \mathrm{mg}$ de estreptomicina e 800 unidades de penicilina. As culturas eram mantidas na estufa a $28^{\circ} \mathrm{C}$.

O isolamento inicial das cepas no LIT foi feito a partir de animais que apresentavam niveis elevados de parasitemia. Estes eram anestesiados com éter e por punção cardiaca em câmara assética retirava-se sangue destinado ao meio de cultura.
Após 8-20 dias seguidos ao semeio do sangue infectado, foi realizado o primeiro repique. Deste primeiro repique, procedia-se novos semeios semanais até se obter um crescimento rico, sendo então iniciados os estudos quantitativos. $O$ inóculo para cada tubo variou entre 5.093-5.950 parasitos/ml; a idade das cepas após o primeiro isolamento foi: $\mathrm{R} 52=67$ dias, $\mathrm{R} 64=63$ dias, $R 65=50$ dias e $M 226=37$ dias. Foram utilizados para cada cepa cinco tubos com cultura. Antes de se proceder às contagens os tubos eram bem homogeneizados e o resultado final corresponddia a média das cinco contagens para cada cepa.

A evolução da população dos tripanosomas, quanto ao seu crescimento numérico e morfogênese, foi acompanhada através da contagem destes em câmara de Neubauer, como está descrito em Camargo ${ }^{\circ}$ (1964) com aumento de $500 \quad(12,5 \times 40)$. Estas contagens eram feitas separando-se as formas amastigotas, epimastigotas e tripomastigotas. A evolução das quatro cepas de $T$. cruzi foi acompanhada durante 13 dias (Tabela 1). Das culturas em diferentes periodos foram feitas lâminas coradas com Giemsa-MayGrunwald e destas lâminas foram tiradas microfotografias com objetiva $\times 100$.

A análise estatística dos dados prendeu-se ao estudo do comportamento das curvas de crescimento da população dos tripanosomas nas quatro cepas e formas. Para cada um dos fenômenos analisados, foi verificada a função que melhor se ajustava às observações e a taxa média de crescimento.

As taxas médias de crescimento foram calculadas a partir de uma média aritmética, média quadrática ou média geométrica dependendo da função ajustante ser uma reta, parábola do $2^{\circ}$ grau, exponencial ou logística. Tais funçōes foram estimadas através do critério dos mínimos quadrados exceto a logistica que foi ajustada a partir do método dos pontos selecionados. 
MELLo, D. A. et al. Crescimento e diferenciação "In vitro" de cepas de Trypanosoma cruzi, isoladas de animais silvestres. Rev. Saúde públ., S. Paulo, 14:569-81, 1980.

T A B E L A 1

Evolução da popilação (número médio de parasitas) de quatro cepas silvestres de T. cruzi, cultivadas em meio LIT.

\begin{tabular}{|c|c|c|c|c|}
\hline \multirow{2}{*}{$\begin{array}{l}\text { Tempo } \\
\text { (horas) }\end{array}$} & \multicolumn{4}{|c|}{ Número de parasitas $/ \mathrm{ml}\left(10^{3}\right)$ por formas } \\
\hline & M226 & R52 & R64 & R65 \\
\hline 0 & 6,6 & 5,1 & 5,1 & 5,8 \\
\hline 10 & $8,3( \pm 4,5)$ & - & - & $7,1( \pm 1,4)$ \\
\hline 24 & $15,8( \pm 5,8)$ & $7,7( \pm 3,2)$ & $8,1( \pm 2,1)$ & $14,4( \pm 3,5)$ \\
\hline 36 & $19,9( \pm 3,5)$ & $8,1( \pm 2,8)$ & $7,6( \pm 2,0)$ & $19,1( \pm 4,3)$ \\
\hline 48 & $32,6( \pm 5,9)$ & $5,1( \pm 2,0)$ & $11,1( \pm 1,8)$ & $24,5( \pm 8,0)$ \\
\hline 60 & $42,3( \pm 4,2)$ & $5,3( \pm 1,7)$ & $12,7( \pm 4,5)$ & $31,7( \pm 7,9)$ \\
\hline 72 & $50,3( \pm 6,5)$ & $7,0( \pm 1,8)$ & $23,0( \pm 2,7)$ & $42,9( \pm 4,9)$ \\
\hline 96 & $77,8( \pm 10,5)$ & $10,1( \pm 2,4)$ & $21,4( \pm 3,4)$ & $57,6( \pm 88)$ \\
\hline 120 & $68,0( \pm 12,7)$ & $11,7( \pm 4,7)$ & $24,6( \pm 4,1)$ & $59,0( \pm 12,0)$ \\
\hline 144 & $80,2( \pm 17,2)$ & $19,7( \pm 7,4)$ & $26,1( \pm 4,0)$ & $78,3( \pm 17,0)$ \\
\hline 168 & $140,2( \pm 25,2)$ & $32,8( \pm 6,7)$ & $29,6( \pm 4,7)$ & $155,2( \pm 235)$ \\
\hline 192 & $149,1( \pm 36,0)$ & $41,7( \pm 16,4)$ & $38,5( \pm 7,7)$ & $141,6( \pm 16,5)$ \\
\hline 216 & $159,2( \pm 27,7)$ & $48,9( \pm 12,7)$ & $46,9( \pm 11,0)$ & $128,8( \pm 15,5)$ \\
\hline 240 & $139,1( \pm 20,4)$ & $124,9( \pm 12,7)$ & $48,8( \pm 14,8)$ & $132,0( \pm 33,8)$ \\
\hline 264 & $144,6( \pm 32,0)$ & $83,5( \pm 17,0)$ & $63,5( \pm 8,4)$ & $108,4( \pm 297)$ \\
\hline 288 & $96,5( \pm 16,5)$ & $82,8( \pm 20,0)$ & $65,7( \pm 6,0)$ & $116,8( \pm 13,5)$ \\
\hline 312 & $102,7( \pm 24,7)$ & $78,4( \pm 14,5)$ & $68,1( \pm 13,8)$ & $96,4( \pm 18,5)$ \\
\hline
\end{tabular}

\section{RESULTADOS}

\section{Análise qualitativa}

As quatro cepas de $T$. cruzi estudadas neste trabalho sempre apresentaram nos repiques iniciais, abundantes aglomerados de formas amastigotas (Fig. 1). Quando a população das cepas atingiam o ponto de saturação no tubo de cultura, praticamente estes aglomerados desapareciam, cedendo lugar aos epimastigotas e tripomastigotas (Fig. 2), embora raros amastigotas livres ainda pudessem ser vistos. Em várias ocasióes foi possivel constatar tripomastigotas como se estivessem em processo de divisão (Fig. 3). Tais aspectos foram observados somente a partir da elevação da população destas formas. A manutenção destas cepas "In vitro" no LIT, por um período de 12-13 meses após o isolamento inicial do $T$. cruzi do sangue, e sucessivos repiques (perfazendo um total

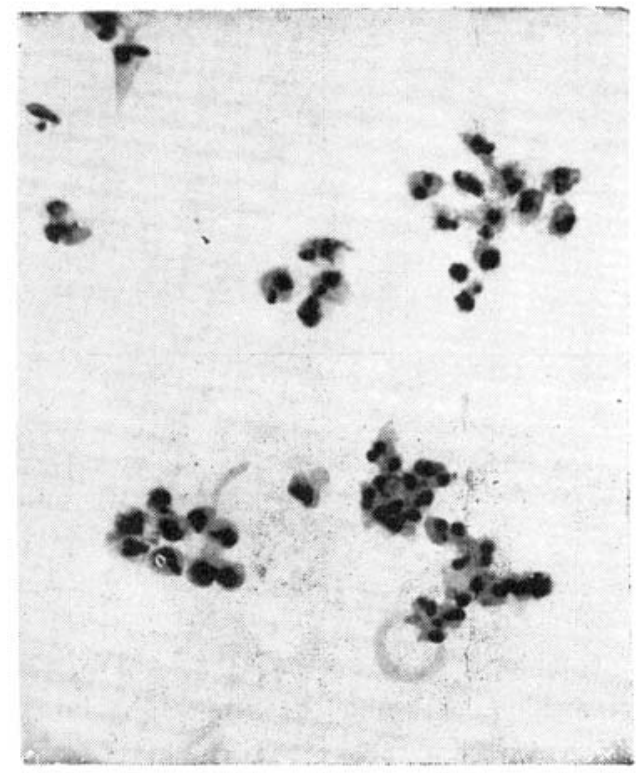

Fig. 1 - Formas amastigotas em aglomerados ou isoladas observadas no meio LIT (x 1.200) 
MELLO, D. A. et al. Crescimento e diferencịaçăo "In vitro" de cepas de Trypanosoma cruzi, isoladas de animais silvestres. Rev. Saúde públ., $\mathrm{S}$. Paulo, 14:569-81, 1980.

de 29), mostrou que havia um decréscimo gradativo e substancial das formas amastigotas.

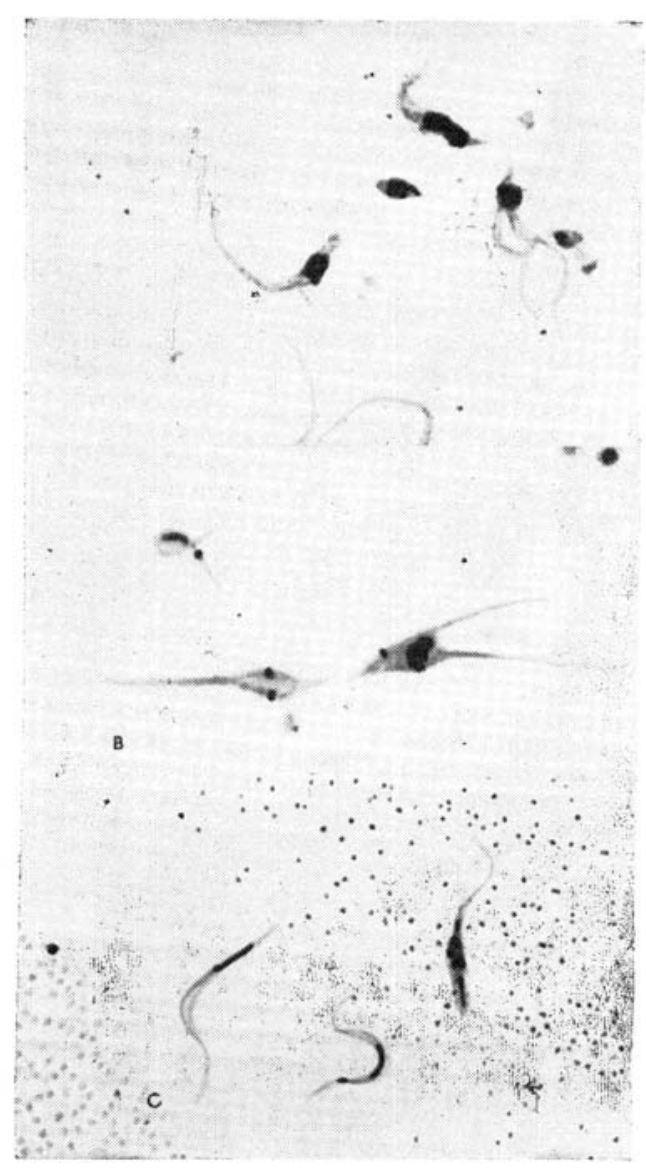

Fig. 2 - Formas observadas no meio IIT $(x$ 1.200) : epimastigotas isoladas (A) ou em divisão; (B) epimastigota em diferenciação e tripomastigota metaciclica (C).

Quanto à sobrevivência no LIT não renovado, os resultados encontrados foram diferentes para cada cepa: R52 morria com 41 dias de manutenção neste meio; R64 morria com 31 dias; R65 com 60 dias; $e$ finalmente $M 226$ com 70 dias.

\section{Análise Quantitativa}

A Tabela 1 apresenta os resultados obtidos sobre os niveis de crescimento no meio LIT das quatro cepas de $T$. cruzi estudadas neste trabalho.

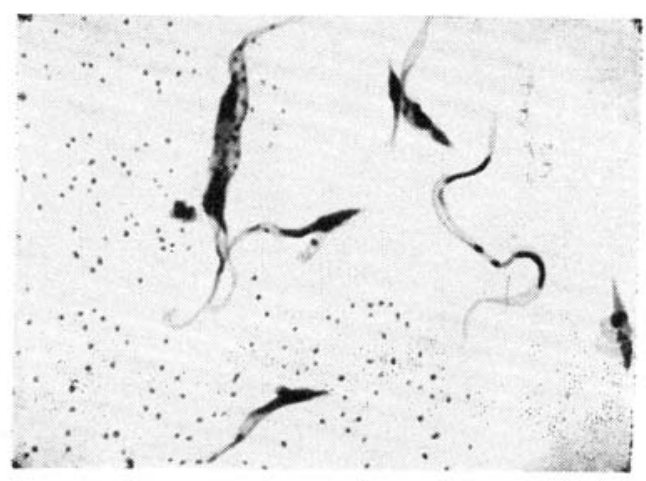

Fig. 3-Forma tripomastigota, sugerindo divisão. observada no mein ILIT $(x$ 1.200).

A cepa que apresentou melhor crescimento numérico total foi a M226, vindo por ordem decrescente a R65, R52 e R64 (Tabela 1 e Fig. 4).

As Tabelas e Figs. 5, 6, 7 e 8 mostram os resultados sobre o crescimento numérico e diferenciação de cada cepa estudada. A produção de amastigotas para a cepa R52 (Tabela 2), durante o período de observação en todo o tempo foi inferior a de epimastigotas e superior as de tripomastigotas até as $264 \mathrm{~h}$. Para a R64 (Tabela 3 ), o crescimento de amastigotas foi superior às epimastigotas e tripomastigotas até as $192 \mathrm{~h}$ se equilibrando numericamente ao final das observações $(288 \mathrm{~h}, 312 \mathrm{~h}$ e 336 h). Com relação às cepas R65 e $M 226$ (Tabelas 4 e 5 ), verificou-se que aquelas mesmas formas tiveram um crescimento numérico maior do que as epimastigotas até as $96 \mathrm{~h}$ e $72 \mathrm{~h}$ respectivamente, sendo entretanto sempre superior as formas tripomastigotas.

Os picos de população das formas e cepas estudadas em relação ao tempo, encontra-se na Tabela 6. 
MELLO, D. A. et al. Crescimento e diferenciação "In vitro" de cepas de Trypanosoma cruzi, isoladas de animais silvestres. Rev. Saúde públ., S. Paulo, 14:569-81, 1980.

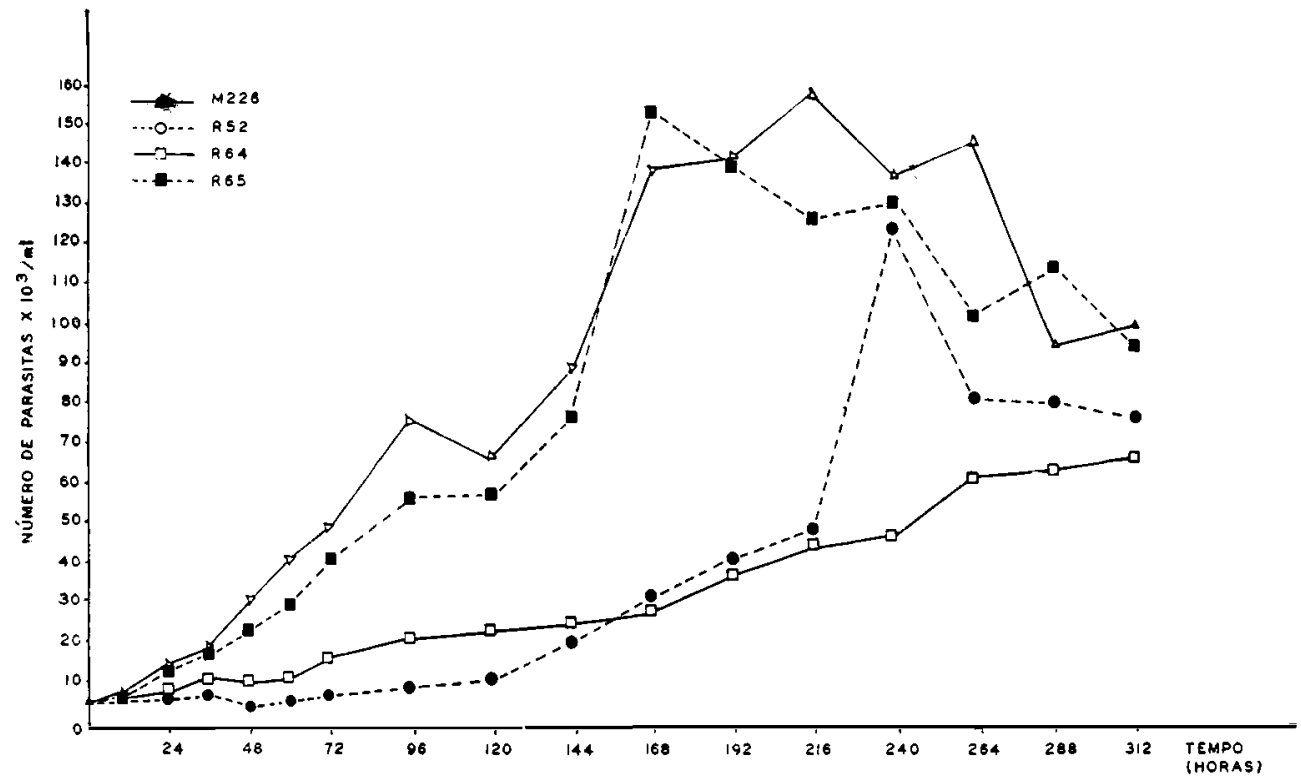

Fig. 4 - Curva de crescimento de cepas silvestres de $T$. cruzi em meio LIT.

TAB E A 2

Crescimento e diferenciaçāo da cepa silvestre de $T$. cruzi, R52 em meio LIT.

\begin{tabular}{|c|c|c|c|}
\hline \multirow{2}{*}{$\begin{array}{l}\text { Tempo } \\
\text { (horas) }\end{array}$} & \multicolumn{3}{|c|}{ Número de parasitas $/ \mathrm{ml} 10^{3}$, por formas } \\
\hline & A & $\mathrm{F}$ & $\mathrm{T}$ \\
\hline 0 & 1.1 & 2,1 & 1,9 \\
\hline 10 & - & - & - \\
\hline 24 & $1.7(=0,6)$ & $3,2( \pm 1,3)$ & $2,8( \pm 1,3)$ \\
\hline 36 & $1,1( \pm 0,6)$ & $3,8( \pm 1,1)$ & $3,2( \pm 1,2)$ \\
\hline 48 & $1,1( \pm 0,5)$ & $3,0( \pm 0,9)$ & $1,1( \pm 0,6)$ \\
\hline 60 & $1,1( \pm 0,3)$ & $3,0( \pm 1,2)$ & $1,2( \pm 0,4)$ \\
\hline 72 & $2,0( \pm 0,5)$ & $4,3( \pm 1,4)$ & $0,7( \pm 0,0)$ \\
\hline 96 & $3.5( \pm 0.9)$ & $5,5( \pm 1,2)$ & $1,1( \pm 0,3)$ \\
\hline 120 & $4,2( \pm 1,3)$ & $5,7( \pm 2,4)$ & $1,8( \pm 1,0)$ \\
\hline 144 & $7,7( \pm 2,0)$ & $9.5( \pm 4.5)$ & $2,5( \pm 1.2)$ \\
\hline 168 & $10,4( \pm 4,2)$ & $16,7( \pm 1,4)$ & $5.7( \pm 1.2)$ \\
\hline 192 & $11.0( \pm 3.4)$ & $25,0( \pm 1,1)$ & $5,7( \pm 2.2)$ \\
\hline 216 & $12,2( \pm 1,9)$ & $30,2( \pm 9,4)$ & $6,5( \pm 1,4)$ \\
\hline 240 & $23,5( \pm 7,5)$ & $80.3( \pm 25.4)$ & $21,1( \pm 1,1)$ \\
\hline 264 & $19,2( \pm 4,6)$ & $45,4( \pm 7.2)$ & $18,9( \pm 7,1)$ \\
\hline 288 & $13,1( \pm 1,2)$ & $44,0( \pm 2.1)$ & $25,7( \pm 6.7)$ \\
\hline 312 & $15.1( \pm 3.5)$ & $39,5( \pm 6,9)$ & $23.8( \pm 4,1)$ \\
\hline
\end{tabular}

$A=$ Amastigotas

$\mathrm{T}=$ Epimastigotas

$\mathrm{T}=$ Tripomastigotas 
MELLO. D. A. et al. Crescimento e diferenciação "In vitro" de cepas de Trypanosoma oruzi, isoladas de animais silvestres. Rer. Saúde pübl, S. Paulo, 14:569-81, 1980.

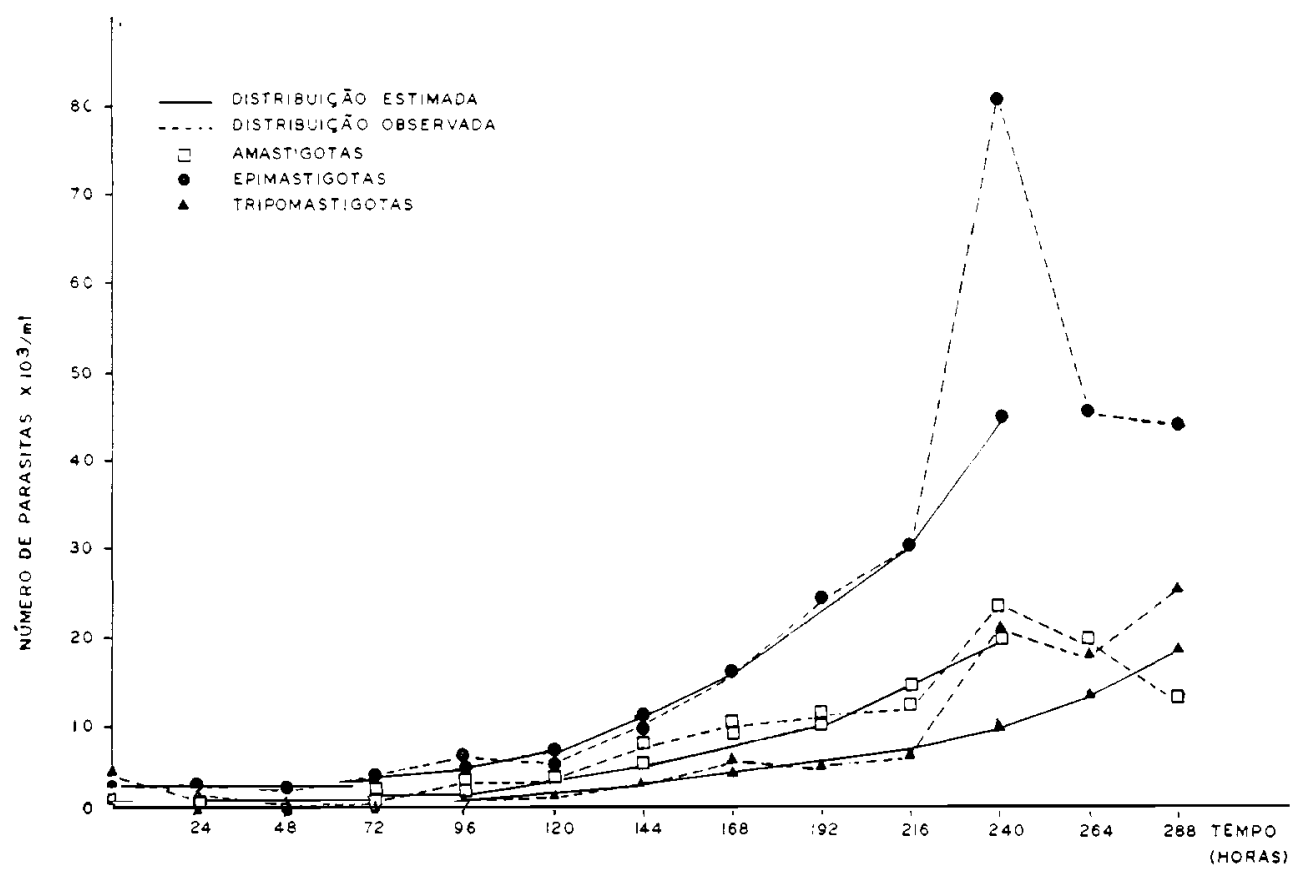

Fig. 5 - Crescimento e diferenciação da cepa silvestre de T. cmai R52, cultivada no meio LIT.

T A B E L A

Crescimento e diferenciação da cepa silvestre de T. cruzi, R64, em meio LIT.

\begin{tabular}{|c|c|c|c|}
\hline \multirow{2}{*}{$\begin{array}{l}\text { Tempo } \\
\text { (horas) }\end{array}$} & \multicolumn{3}{|c|}{ Número de parasitas $/ \mathrm{ml}\left(10^{3}\right)$ por formas } \\
\hline & $\mathrm{A}$ & $\mathrm{E}$ & $\mathrm{T}$ \\
\hline 0 & 2.9 & 1,2 & 1.0 \\
\hline 10 & - & - & - \\
\hline 24 & $4,51 \pm 1,2)$ & $2.5( \pm 0.6)$ & $1.1( \pm 0.4)$ \\
\hline 36 & $3.6( \pm 0.6)$ & $2,9( \pm 0,7)$ & $1,1( \pm 0,7)$ \\
\hline 48 & $6.7( \pm 0.9)$ & $3,4( \pm 0.6)$ & $1,0( \pm 0.4)$ \\
\hline 60 & $8,3( \pm 3,0)$ & $3,4( \pm 1,0)$ & $1.0( \pm 0.4)$ \\
\hline 72 & $10,6( \pm 1,4)$ & $5,8( \pm 0,9)$ & $0.6( \pm 0,3)$ \\
\hline 96 & $14.7( \pm 1,6)$ & $4.9( \pm 1,0)$ & $1.8( \pm 0.8)$ \\
\hline 120 & $15,3( \pm 2,5)$ & $6,3( \pm 0.9)$ & $2.4( \pm 0.5)$ \\
\hline 144 & $14,4( \pm 1,8)$ & $8,5( \pm 1,2)$ & $3.2( \pm 1,0)$ \\
\hline 168 & $15.8( \pm 1.3)$ & $10,8( \pm 1.7)$ & $3.0( \pm 1.6)$ \\
\hline 192 & $20.3( \pm 4,0)$ & $13,6( \pm 2,6)$ & $4.6(i=1.0)$ \\
\hline 216 & $18,0( \pm 2.5)$ & $18.1( \pm 3.6)$ & $10.8( \pm 4.8)$ \\
\hline 240 & $20,0( \pm 3,7)$ & $19,0( \pm 1,4)$ & $9.81 \pm 2.9)$ \\
\hline 264 & $19,8( \pm 1,6)$ & $22,2( \pm 3.5)$ & $21.5( \pm 4.0)$ \\
\hline 288 & $22,7( \pm 3,6)$ & $21,3( \pm 2,4)$ & $21,7( \pm 2,4)$ \\
\hline 312 & $20,9( \pm 3,6)$ & $21,5( \pm 1,4)$ & $25,7( \pm 8,8)$ \\
\hline
\end{tabular}

$A=$ Amastigotas

$\mathrm{E}=$ Epimastigotas

$T=$ Tripomastigotas 
MELLO, D. A. et al. Crescimento e diferenciação "In vitro" de cepas de Trypanosoma cruzi, isoladas de animais silvestres. Rev. Saude publ. S. Paulo, 14:569-81, 1980.

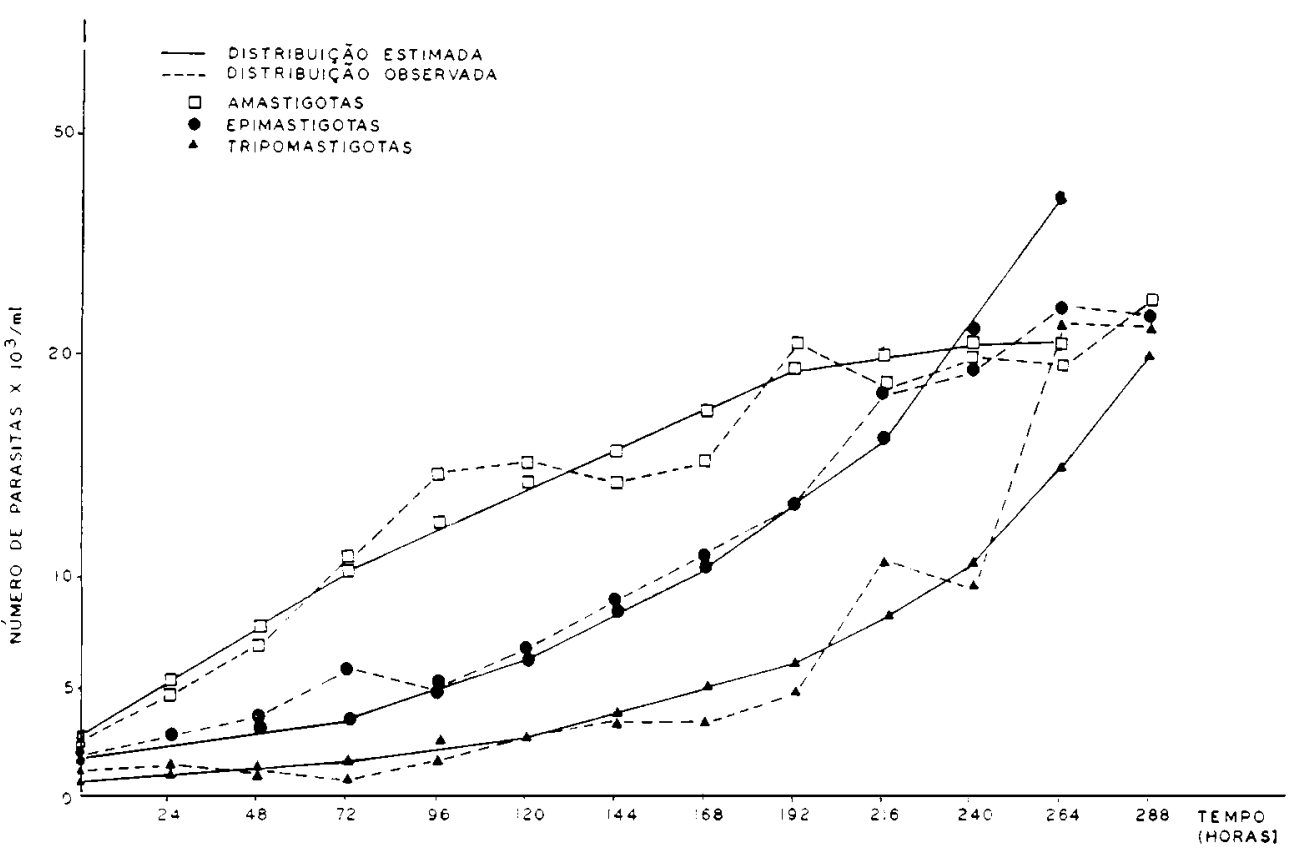

Fig. 6 - Crescimento e diferenciação da cepa silvestre de T. crizi R64, cultivada no meio LIT.

TABELA 4

Crescimento e diferenciação dà cepa silvestre de $T$. cruzi, R65, em meio LIT.

\begin{tabular}{|c|c|c|c|}
\hline \multirow{2}{*}{$\begin{array}{l}\text { Tempo } \\
\text { (Jiortas) }\end{array}$} & \multicolumn{3}{|c|}{ Número de parasitas $/ \mathrm{ml}\left(10^{3}\right)$ por formas } \\
\hline & $A$ & $\mathrm{E}$ & $\mathrm{T}$ \\
\hline 0 & 1,7 & 3.9 & 0.2 \\
\hline 10 & $2.4( \pm 0.3)$ & $4.4( \pm 1,0)$ & $0,3( \pm 0,1)$ \\
\hline 24 & $7,0( \pm 2,0)$ & $6.4( \pm 0.8)$ & $1.0( \pm 0.7)$ \\
\hline 36 & $9,8( \pm 2.4)$ & $8,5( \pm 1,6)$ & $0.8(=0.3)$ \\
\hline 48 & $13,3( \pm 4.5)$ & $10,6( \pm 3,2)$ & $0,6( \pm 0,4)$ \\
\hline 60 & $17,4( \pm 4,3)$ & $13.0( \pm 2,7)$ & $1.3( \pm 0.9)$ \\
\hline 72 & $21,8( \pm 1.9)$ & $19,7( \pm 2,3)$ & $1.4( \pm 0.6)$ \\
\hline 96 & $28,9( \pm 6,0)$ & $26,9( \pm 2,0)$ & $1.8( \pm 0.8)$ \\
\hline 120 & $22,8( \pm 8,9)$ & $33,3( \pm 2,2)$ & $2.9( \pm 0,9)$ \\
\hline 144 & $13,8( \pm 3,7)$ & $60.9( \pm 10,0)$ & $8.6( \pm 3,2)$ \\
\hline 168 & $54,4( \pm 11,0)$ & $80.0( \pm 9.7)$ & $20.8( \pm 2,8)$ \\
\hline 192 & $56.0( \pm 5.0)$ & $63.2( \pm 1,4)$ & $22.41 \pm 10.0$ \\
\hline 216 & $40.8( \pm 6,3)$ & $64,8( \pm 4,1)$ & $23.2( \pm 5.01$ \\
\hline 240 & $37,6( \pm 15,4)$ & $64,0( \pm 8,4)$ & $30,4( \pm 10,0)$ \\
\hline 264 & $28,8( \pm 10,5)$ & $49.0( \pm 8.4)$ & $25,6( \pm 10.8)$ \\
\hline 288 & $30,4( \pm 8,4)$ & $60,0( \pm 0.0)$ & $26,4( \pm 8,3)$ \\
\hline 312 & $32,0( \pm 5.0)$ & $39.2( \pm 8.4)$ & $25.2( \pm 5.0)$ \\
\hline
\end{tabular}

$\mathrm{A}=$ Amastigotas

$\mathrm{E}=$ Epimastigotas

$\mathrm{T}=$ Tripomastigotas 
MELLO, D. A. et al. Crescimento e diferenciaģāo "In vitro" de cepas de Trypanosoma cruzi, isoladas de animais silvestres. Rev. Saúde públ., S. Paulo, 14:569-81, 1980.

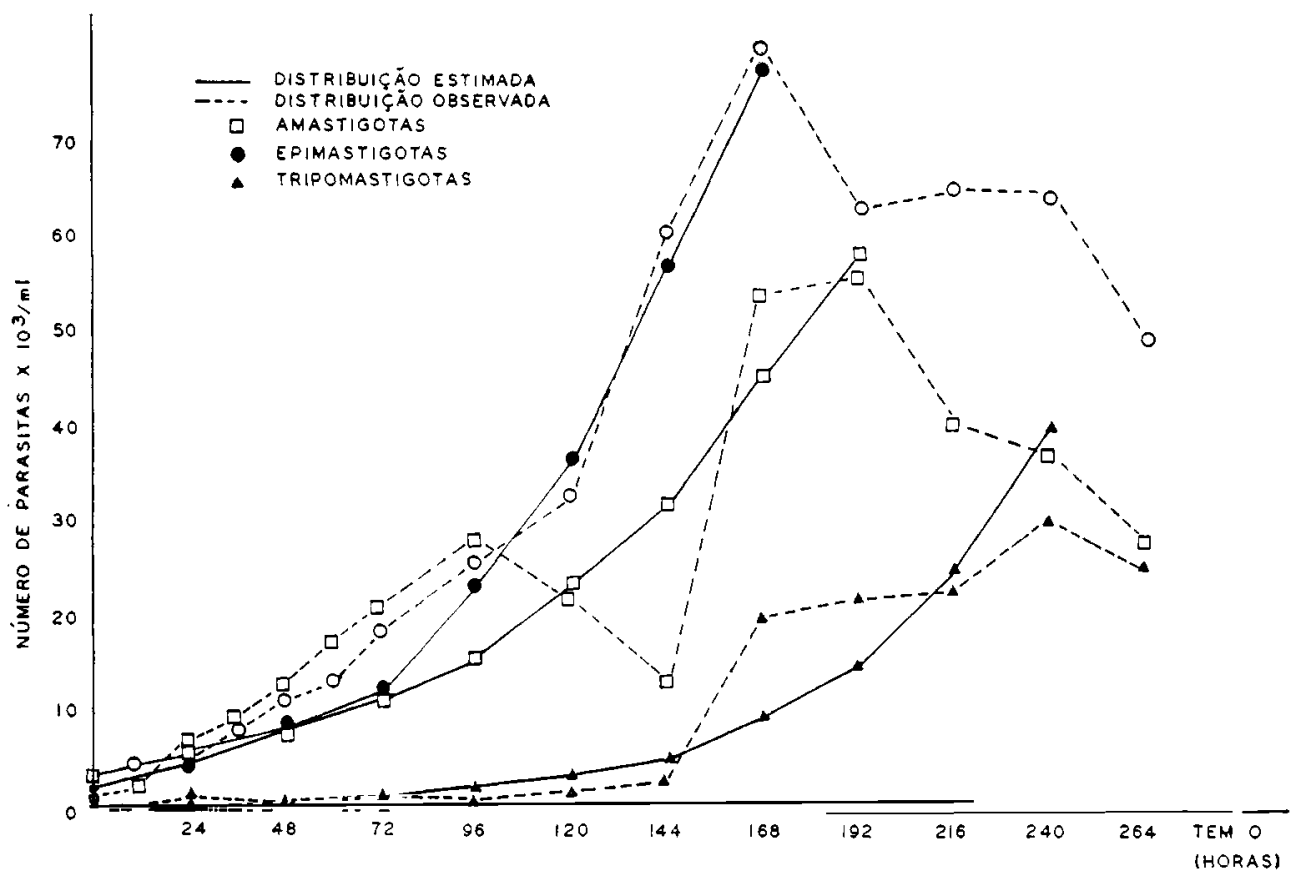

Fis. 7 -- Crescimento e diferenciação da cepa silvestre de $T$. cruzi R65, cultivada en meio LIT.

TA B E L A 5

Crescimento e diferenciação da cepa silvestre de T. cruzi, M226, em meio LIT.

\begin{tabular}{|c|c|c|c|}
\hline \multirow{2}{*}{$\begin{array}{l}\text { Tempo } \\
\text { (horas) }\end{array}$} & \multicolumn{3}{|c|}{ Número de parasitas $/ \mathrm{ml}\left(10^{3}\right)$ por formas } \\
\hline & $A$ & $\mathrm{E}$ & $\mathrm{T}$ \\
\hline 0 & 2,2 & 3,6 & 0,2 \\
\hline 10 & $2,0( \pm 1,4)$ & $5,1( \pm 1,0)$ & $1,2( \pm 2,0)$ \\
\hline 24 & $7,2( \pm 2,8)$ & $7,5( \pm 2,6)$ & $1.1( \pm 0.4)$ \\
\hline 36 & $9,8( \pm 1,2)$ & $9,1( \pm 1,6)$ & $1,0( \pm 0.7)$ \\
\hline 48 & $17,5( \pm 2,4)$ & $13,4( \pm 2,3)$ & $1.7( \pm 1,1)$ \\
\hline 60 & $23,1( \pm 3,0)$ & $17,9( \pm 0,6)$ & $1,3( \pm 0,5)$ \\
\hline 72 & $26,6( \pm 2,7)$ & $22,0( \pm 3,0)$ & $1.7(-50,8)$ \\
\hline 96 & $37,5( \pm 4,9)$ & $37,6( \pm 5,0)$ & $2,7( \pm 0,6)$ \\
\hline 120 & $19.9( \pm 5.8)$ & $44,6( \pm 3,3)$ & $3,5( \pm 0,5)$ \\
\hline 144 & $15,7( \pm 5,0)$ & $67,2( \pm 10.1)$ & $7,3( \pm 2,1)$ \\
\hline 168 & $43,7( \pm 13,9)$ & $74,9( \pm 4,3)$ & $21,6( \pm 7,0)$ \\
\hline 192 & $49,3( \pm 11,2)$ & $79,7( \pm 15,0)$ & $20,1( \pm 4,0)$ \\
\hline 216 & $51,1(=6.9)$ & $75.5( \pm 15,8)$ & $32,6( \pm 5,0)$ \\
\hline 240 & $48,9( \pm 9,7)$ & $59,5( \pm 6.0)$ & $30,7( \pm 4,9)$ \\
\hline 264 & $42,0( \pm 11,3)$ & $66,6( \pm 12,0)$ & $36,0( \pm 9,8)$ \\
\hline 288 & $36,0( \pm 4,1)$ & $33,1( \pm 7,0)$ & $27,3( \pm 5,2)$ \\
\hline 312 & $30.2( \pm 7.9)$ & $48,0( \pm 9,9)$ & $24,5( \pm 6.9)$ \\
\hline
\end{tabular}

$\mathrm{A}=$ Amastigotas
$\mathrm{E}=$ Epimastigotas
$\mathrm{T}=$ Tripomastigotas


MELLO, D. A. et al. Crescimento e diferenciação "In vitro" de cepas de Trypanosoma cruzi, isoladas de animais silvestres. Rev. Saúde públ., S. Paulo. 14:569-81, 1980.

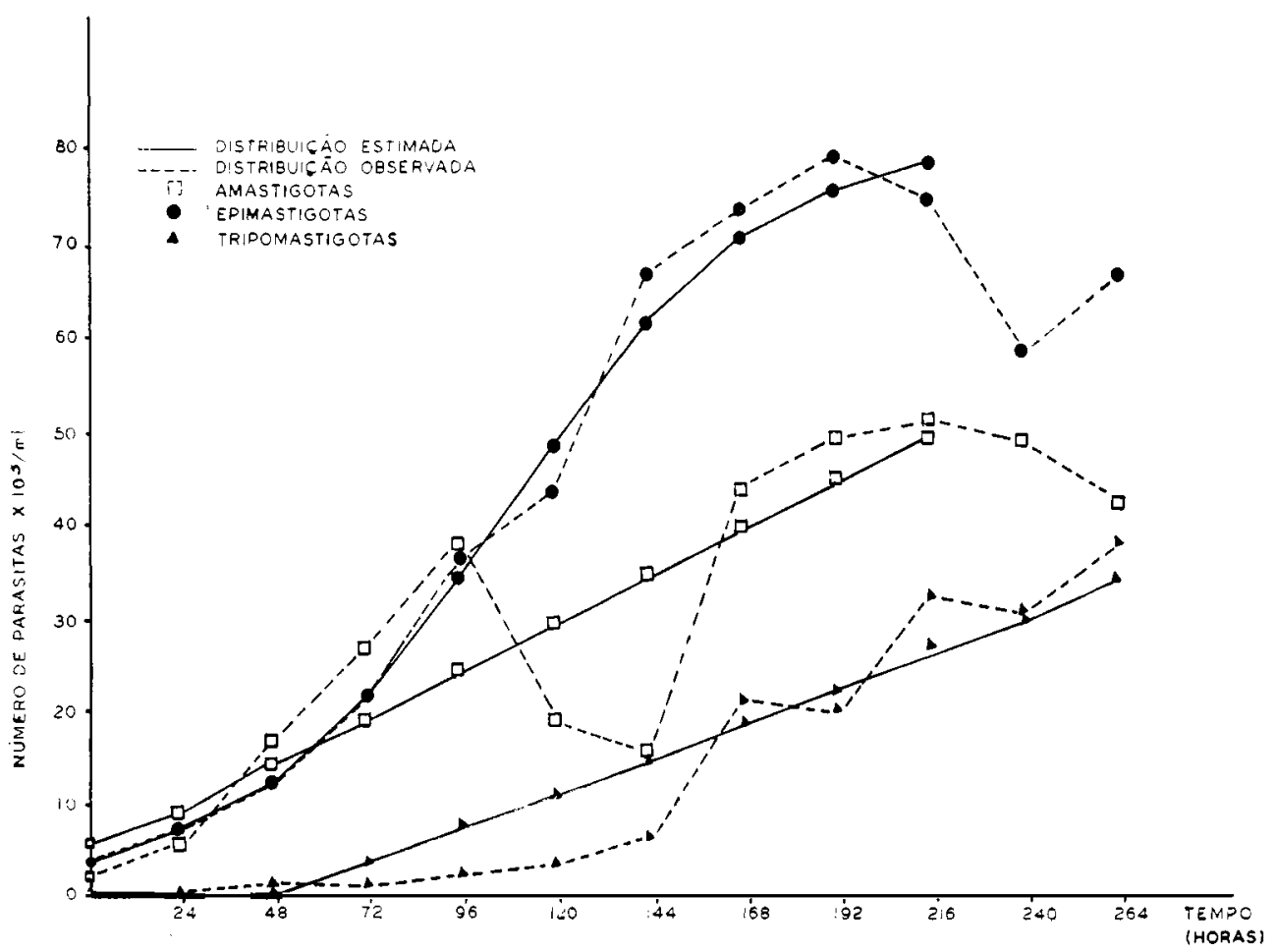

Fig. 8 - Crescimento e diferenciação da cepa silvestre de T. orusi M226, cultivada no melo LIT.

T A B EL 6

Picos de população das formas amastigotas, epimastigotas e tripomastigotas, das quatro cepas silvestres de $T$, cruai

\begin{tabular}{cc|c}
\hline Cepas e formas & Picos de população \\
(horas)
\end{tabular}

As curvas de crescimento para cada forma e cepa estão representadas nas Figs. $5,6,7$ e 8 . As taxas de crescimento e tipo de curvas obtidas estão apresentadas na Tabela 7.

Enquanto as cepas R52 e R65 apresentaram um crescimento exponencial para as três formas, a cepa R64 só o apresentou para as epimastigotas e tripomastigotas. A forma amastigota desta cepa apresentou um crescimento representado por uma parábola do segundo grau. Para cepa M226 as formas amastigotas, epimastigotas e tripomastigotas mostraram, respectivamente, um crescimento representado por uma reta, uma logística e uma reta.

A maior taxa média de crescimento das formas amastigotas deu-se na cepa M226, seguida pela R65, R52 e R64. Para as epimastigotas a cepa $R 65$ foi a que apre- 
MELLO, D. A. et al. Crescimento e diferenciaçăc "In vitro" de cepas de Trypanosoma cruzi, isoladas de animais silvestres. Rer. Saúde puibl., S. Paulo. 14:569-81, 1980.

TABELA 7

Tipos de curras e taxas de crescimento obtidas para quatro cepas silvestres de $T$. crual.

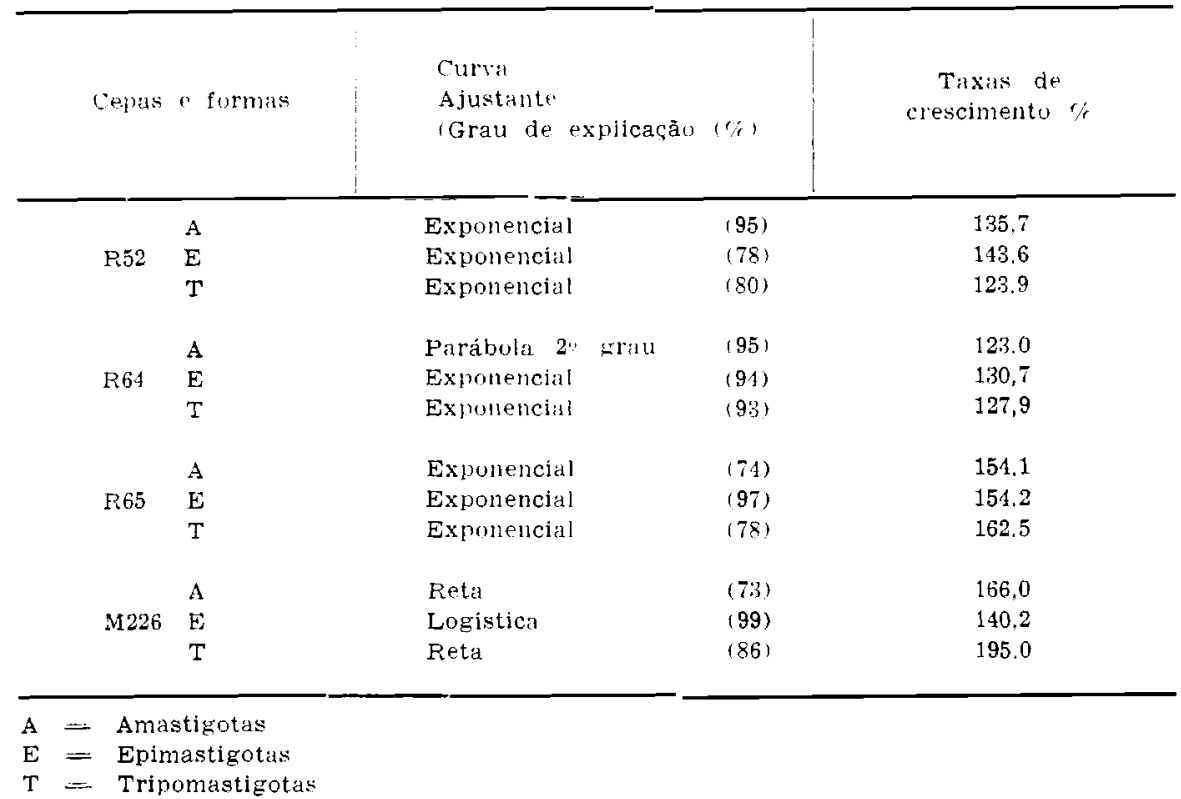

sentou a maior taxa media de crescimento, seguindo-se as cepas R52, M226 e R64. Finalmente, as tripomastigotas, que apresentaram a maior taxa média de crescimento foram as da cepa M226, seguindo-se as R65, R64 e R52.

\section{COMENTARIOS E CONCLUSOES}

Norman e col.\% (1959) estudando sete cepas de $T$. cruzi isoladas de animais silvestres en meio bifásico, verificaram que todas elas apresentavam formas amastigotas yue apareciam entre o 4! e 100 dia após incubação. $O$ número destas formas dependia, segundo estes autores, da quantidade do inóculo. A partir do $14^{\circ}$ dia as culturas apresentavam as formas epimastigotas. Os tripomastigotas só eram observados em baixo número, após várias transferências e em culturas com 28 dias de idade. Das sete cepas estudadas, apenas uma, isolada de Procyonlotor, apresentou mudanças de comportamento durante os experimentos.

Brener e Chiari" (I965), an estudar " comportamento "In vitro" no meio LIT, de quatro cepas isoladas de casos humanos e três isoladas de Triatoma infestans, verificaram diferentes comportamentos. As cepas isoladas de triatomineo cujas formas sanguicolas eram bem largas deram origem nos repiques iniciais a massas coloniais de amastigotas puras ou com epimastigotas. Estas cepas, após repiques a cada 10 dias por um período de dois meses, apresentavam gradual desaparecimento das amastigotas, sendo substituidas por epimastigotas. Nas cepas de origem humana, cujas formas sanguicolas eram predominantemente delgadas ( $Y$ e Berenice), as amastigotas rapidamente desapareciam e após dez dias de incubação só havia epimastigotas livres ou em tosetas. As outras duas cepas, de origem humana ( $A B C$ e PNM), com formas sanguicolas largas, embora apresentassem 
MELLo, D. A. et al. Crescimento e diferenciasăo "In vitru" de cepas de Trypanosoma cruzi, isoladas de animais silvestres. Rev. Saude públ., S. Paulo. 14:569-81, 1980.

"In vitro" epimastigotas em dominância, no entanto raras massas de amastigotas poderiam ainda ser encontradas. Concluiram esses autores que a presença ou ausência das amastigotas estaria relacionada diretamente com características intrinsecas das cepas.

Ribeiro e Barretto 1ti (1975) estudaram comportamento "In vitro" no meio Warren, de 10 cepas de $T$. cruzi de diferentes origens comparando-o com infecções experimentais em camundongos. Evidenciaram estes autores comportamentos diferentes, que levou-os a inserir estas cepas em três grupos: 1 (uma amostra isolada de $D$. azarae e uma de Triatoma infestans) as formas sanguicolas dominantes nos camundongos infectados eram largas; na cultura as colonias de amastigotas persistiam após quatro subculturas e praticamente desapareciam dando lugar às formas flageladas; Il (duas amostras de Tadarida laticaudata e duas humanas, Indio e $Y$ ) nestas raramente encontravam-se amastigotas; 111 - (três amostras de roedores Akodon sp. e Sciurus sp. e uma de Cebus apella) morfologia sanguicola heterogênea; as culturas recentes apresentavam amastigotas grupadas ou isoladas, as quais desapareciam paulatinamente para dar lugar só às formas epimastigotas e tripomastigotas.

Os resultados de Ribeiro e Barretto ${ }^{16}$ (1975) apresentaram concordância em parte com os de Brener e Chiari * (1965) no que se refere aos aspectos da morfologia das formas sanguicolas e o comportamento "In vitro", discordando entretanto no que se refere a patogenicidade.

Ao se analisar do ponto de vista qualitativo, os dados obtidos neste trabalho, veri-. fica-se que o comportamento das amastigotas no LIT foi homogêneo para as quatro cepas estudadas. Todas apresentaram aglomerados numerosos de formas amastigotas nas culturas jovens, os quais diminuiam progressivamente nos repiques suces. sivos para ceder lugar às epimastigotas e tripomastigotas (Figs. 1 e 2). Chama atenção o encontro das tripomastigotas aos pares conı se estivessem en divisão (Fig. 3). Achado similar está relatado no trabalho de Camargo' (1964). As quatro cepas tinham formas sanguicolas predominantemente largas sendo pouco patogênicas para camundongos e Calomys callosus (Mello e col.1n 1979). Pode-se concluir portanto, referindo-se ao comportamento das amastigotas, ao tipo das formas sanguicolas e à patogenicidade, que os resultados aqui obtidos não seguem os mesmos padrões daqueles encontrados por Brener e Chiari : (1965) e Ribeiro e Barretto 1ti (1975).

Camargo ${ }^{6}$ (1964), estudando o comportamento de cepa $Y$ no meio $\mathrm{LIT}$, verificou que havia uma fase de crescimento que correspondia a um período de quatro dias. O número de metaciclicos se desenvolvia no final da fase exponencial e atingia o maior número na fase estacionária. Quando este autor mantinha as culturas com indice logaritimo permanente obtido em subculturas, o pico metaciclico jamais ocorria. A fase logaritimica caracterizava-se por uma predominància de epimastigotas.

Castellani e col.' (1967) mostraram que a diferenciação de epimastigotas em tripomastigotas seria estimulada por acumulação de ácidos orgânicos no meio, no final do crescimento exponencial. Esses resultados foram confirmados por Pan ${ }^{14}$ (1971), ao estudar o comportamento de cepa Brasil em dois meios diferentes com três variações de temperatura $\left(24,5^{\circ} \mathrm{C}, 29,5^{\circ} \mathrm{C}\right.$ e $\left.35^{\circ} \mathrm{C}\right)$. Concluiu esse autor que a composição do meio é que seria importante na produção numérica e diferenciação do parasito.

Chiari 9 (1974) analisou crescimento e diferenciação nos meios LIT e HIL, das cepas humanas de $T$. cruzi, Y e MR, mantidas "In vitro" por diferentes períodos de tempo após isolamento do hospedeiro. Estas cepas haviam sido estudadas anteriormente por Brener e Chiari ' (1963), Camargo " (1964) e Brener e Chiari' (1965). No trabalho de Chiari ii (1974) os resultados encontrados não mostraram diferenças significantes quanto ao crescimento das 
MELLO, D. A. et al. Crescimento e diferenciação "In vitro" de cepas de Trypanosoma cruzi, isoladas de animais silvestres. Rev. Saúde públ., 8. Paulo, 14:569-81, 1980.

duas cepas guardadas por longo tempo. As modificaçōes observadas referianl-se a metaciclogênese.

Do ponto de vista quantitativo, os resultados aqui obtidos mostraram diferenças de crescimento estatisticamente significantes para cada cepa e forma (Tabelas 2, 3, 4, 5 e 7). Comparar estes resultados com aqueles de Camargo' (1964) e Chiari : (1974) ell relação aos aspectos quantitativos, seria discrepante uma vez que as cepas aqui estudadas foran isoladas recentemente do hospedeiro vertebrado (37-67 dias). Comentários entretanto poderiam ser conduzidos no que se refere ao tipo de crescimento obtido com estas cepas de T. cruzi. Como mostra as Figs $5,6,7 \mathrm{e}$ 8 e a Tabela 7, as curvas de crescimento nem sempre foram as mesmas para todas as formas e cepas. Embora as cepas R52 e R65 tenham tido diferenças de crescimento distintas, evoluiram entretanto dentro de un mesmo padrão, i.e., as curvas de crescimento obtidas foram do tipo exponencial (Tabela 6 e Figs. 5 e 7). As formas amastigotas da cepa R64 evoluiram segundo uma parábola do segundo grau, enquanto as epimastigotas e tripomastigotas segundo uma exponencial (Tabela 6, Fig. 6). As formas amastigotas e tripomastigotas da cepa M226 deram una reta enquanto as epimastigotas deram una logistica.

Os resultados obtidos na pesquisa ayui realizada levaram a conclusão de que as quatro cepas de $T$. cruzi estudadas têm conportamento distintos. Estes resultados devem no entanto ser encarados considerando que: estas cepas foran isoladas de hospedeiros vertebrados silvestres; foram estudadas logo depois de isolamento "In vitro"; têm pouca patogenicidade para camundongos (Mello e col.10 1979), e as formas sanguícolas são predominantemente largas.

\section{RSPUB9/531}

MELLO, D. A. et al. /Growth and differentiation "In vitro" of strains of Trypanosoma cruzi isolated from wild animals.J Rev. Saúde públ., S. Paulo, 14: $569-81,1980$.

ABSTRACT: In the course of this work, four strains of sylvatic Trypanosoma cruzi were studied in regard to their growth and differentiation in LIT medium. R52, R64, and R65 were isolated from individual Didelphis albiventris (Marsupialia) and M226 from Calomys callosus (Rodentia). The evolution of the numerical incresase and morphogenesis of the trypanosome populations in the culture was observed at regular intervals during a period of 312 hours. Differential counts were made of amastigotes, epimastigotes, and trypomastigotes in Neubauer chambers. Results showed that the four strains had characteristic behavior patterns. M226 increased the most and was followed by R65, R52, and then R64.

UNITERMS: Trypanosoma cruzi, wild strains. Didelphis albiventris. Calomys callosus. 
MELLO, D. A. et al. Crescimento e diferenciação "In vitro" de cepas de Trypanosoma cruzi, isoladas de animais silvestres. Rev. Saúde públ., S. Paulo, 14:569-81, 1980.

\section{REFERENCIAS BIBLIOGRAFICAS}

1. ANDRADE, S. Tentative for growping different Trypanosoma cruzi strains in some types, Rev. Inst. Med. trop. S. Paulo, 18:140-1, 1976.

2. BRENER. Z. Comparative studies of different strains of Trypanosoma cruzi. Ann. trop. Med. Parasit., 59:19-26. 1965.

3. BRENER, Z. Intraspecific variations in. Trypanosoma cruzi: two types of parasite populations presenting distinct characteristics. Washington, D. C. Pan American Health Organization, 1977. p. 11. (PAHO-Scient. Publ., 347).

4. BRENER, Z. \& CHIARI, E. Aspects of early growth of different Trypanosoma cruzi strains in culture medium. J. Parasit., 51:922-6, 1965.

5. BRENER, Z. \& CHIARI, E. Variaçōes morfológicas observadas em diferentes amostras de Trypanosoma cruzi. Rev. Inst. Med. trop. S. Puulo, 5:220-4, 1963.

b. CAMARGO, E. P. Growth and differentiation in Trypanosoma cruzi. I. Origin of metacyclic trypanosoma in liquid media. Rev. Inst. Med. trop. S. Paulo, 6:93-100, 1964 .

7. CASTELlani, $O$, et al. Differentiation of Trpanosoma cruzi in culture. J. Protozool., 14:447-51, 1967.

8. CHAGas, C. Nova Tripanossomíase humana. Estudos sobre a morfologia e ciclo evolutivo do Schizotrypanum cruzi $\mathrm{n}$. gen, n. sp.; Agente etiológico de nova entidade morbida do homem. Mem. Inst. Oswaldo Cruz, 1:159-217, 1909.

9 CHIARI, E. Growth and differentiation of Trypanosoma cruzi culture forms kept in laboratory for different periods of time. Rev. Inst. Med. trop. S. Paulo, $16: 81-7, \quad 1974$

10. MELLO, D. A. et al. Alguns aspectos do comportamento de cepas silvestres de Trypanosoma cruzi em camundongos e Calomys callosus (Rodentia). Rev. Saúde públ., S. Paulo, 13:314-25, 1979.

11. MUNIZ, J. \& FREITAS. G. Realização "In vitro" do ciclo do $S$. cruzi no vertebrado, em meios de caldo líquido peritoneal. Rev. bras. Biol., 6:467-84, 1946.

12. NOBLE, E. R. The morphology and life cycles of trypanosomes. Quart. Rev. Biol., 30:1-28, 1955 .

13. NORMAN, L. et al. Morphology and virulence of Trypanosoma cruzi - like hemoflagellates isolated from wild mammals in Georgia and Florida. $J$. Parasit., 45:457-63, 1959.

14. PAN, C. T. Cultivation and morphogenesis of Trypanosoma cruzi in improved liquid media. J. Protozool., 18:556-60, 1971.

15. PICK, F. Siir un nouveau milieu au sang permettant la transmission rapide de $T$. cruzi (formes sanguines et metacyclique) en forme leismaniènes. Bull. Soc. Path. exot., 50:217-19, 1959.

16. RIBEIRO, R. D. \& BARRETO, M. P. Estudos sobre reservatórios e vetores silvestres do Trypanosoma oruzi LXI. Aspectos do comportamento de amostras diversas de $T$. oruzi em cultura. Rev. bras. Biol., 35:301-7, 1975.

Recebido para publicacão em 02/09/1980 Aprovado para publicação em 22/09/1980 